Cahiers d'études italiennes

\title{
Éthique de la différence dans Il padre e lo straniero de Giancarlo De Cataldo
}

\section{Brigitte Le Gouez}

\section{OpenEdition}

Journals

Édition électronique

URL : http://journals.openedition.org/cei/922

DOI : $10.4000 /$ cei.922

ISSN : 2260-779X

\section{Éditeur}

UGA Éditions/Université Grenoble Alpes

\section{Édition imprimée}

Date de publication : 15 mai 2008

Pagination : 183-193

ISBN : 978-2-84310-121-2

ISSN : $1770-957$

Référence électronique

Brigitte Le Gouez, "Éthique de la différence dans /l padre e lo straniero de Giancarlo De Cataldo » Cahiers d'études italiennes [En ligne], 7 | 2008, mis en ligne le 15 novembre 2009, consulté le 19 avril 2019. URL : http://journals.openedition.org/cei/922 ; DOI : 10.4000/cei.922 


\title{
ÉTHIQUE DE LA DIFFÉRENCE DANS \\ IL PADRE E LO STRANIERO DE GIANCARLO DE CATALDO
}

\author{
Brigitte Le Gouez
}

Université de la Sorbonne Nouvelle-Paris 3

Ce sont ici des notes de lecture que nous proposons, plus qu'une analyse véritablement aboutie. En effet, Il padre e lo straniero de Giancarlo De Cataldo est un roman qui peut susciter quelque perplexité. Le texte pourrait lui-même apparaître comme inabouti, à moins qu'il ne manifeste, précisément, une volonté d'inachèvement. Ce sont ces questionnements du lecteur que nous avons résolu d'exposer ici. Le récit oscille entre "giallo" et "noir», double coloration à laquelle nous avait déjà habitués Giancarlo De Cataldo, juge en Cour d'Assise et auteur de romans dont le plus célèbre est sans doute Romanzo criminale $^{1 *}$, sorte d'épopée de la Banda della Magliana, bien connue des romains dans les années 80. De ce volumineux récit de 600 pages a été tiré un film, sorti en Italie en septembre 2005. Probablement en raison du succès du premier ou à cause de l'attente suscitée par le projet cinématographique, Il padre e lo straniero, court roman de 147 pages, édité par e/o en 2004 après parution en feuilleton dans le journal Il Manifesto, a relativement peu retenu l'attention de la critique.

Dans les vingt dernières années, alors que prenait forme une Italie multiethnique et pluriconfessionnelle, le personnage de l'étranger «extracomunitario" n'a fait qu'une entrée discrète dans le champ littéraire. Il est pourtant une des figures-types de la société des années 90 et du début du XXI siècle. Nous ne parlerons pas ici de la littérature dite "migrante», écrite par ces écrivains immigrés en Italie qui préferent maintenant se définir comme "Italiens par vocation». C'est une réalité littéraire apparue dans les années 90 sur laquelle nous nous sommes penchée en d'autres lieux ${ }^{2}$. En parallèle, il n'est pas moins intéressant de mener une recherche sur la figure de l'étranger dans la littérature contemporaine écrite par des auteurs italiens de naissance. L'étranger est, en effet, une figure éminemment propice à tous les fantasmes. C'est, notamment, un "suspect» de 
choix pour le roman policier. L'autre - plus encore s'il est culturellement différent - se présente dans son opacité au regard de l'enquêteur dont le rôle est précisément de rétablir la transparence et la lisibilité du réel. Sandrone Dazieri utilise cette figure d'étranger «suspect numéro un » dans une des enquêtes de la série des "Gorilla». Dans La cura del Gorilla 3 (2001), l'opacité du mystère se concentre autour de trois personnages albanais: victimes ou assassins? martyrs ou bourreaux? jusqu'à la fin et même après, d'ailleurs, leur statut reste ambigu.

Parmi les premiers écrivains contemporains italiens à avoir pris acte des transformations sociales de l'Italie devenue terre d'accueil, il faut citer Andrea Camilleri. La communauté tunisienne de Mazara del Vallo, cas de figure bien connu des sociologues et géographes travaillant sur l'immigration en Italie, alors qu'elle attirait peu l'attention des gens de Lettres, n'avait pas échappé à Camilleri, qui y fait référence, en 1996, dans Il ladro di merendine ${ }^{4}$. D'autres romans, encore antérieurs, pourraient être cités: par exemple, Una ignota compagnia (1992) de Giulio Angioni, dont Franco Manai nous entretient dans le présent ouvrages. Comme chez Camilleri, on peut considérer que cet intérêt manifesté pour le personnage de "l'étranger» comme composante désormais intégrante du panorama social italien est relativement précurseur. Dans Il giro di boa $(2003)^{6}$, Camilleri revient à la question de l'immigration à travers l'évocation de la difficile question des traversées maritimes des migrants clandestins. Alors que les naufrages en Méditerranée sont devenus une tragédie périodique, pour ne pas dire "habituelle", et qui ne suscite même plus guère d'émotion excessive, Camilleri est un des rares écrivains à avoir abordé le sujet. De Cataldo peut aussi faire figure de précurseur dans la réflexion qu'il mène sur les dérives de nos sociétés post-industrielles. Dès 1989, dans Nero come il cuore, paru dans la collection des "gialli » Mondadori, il traite d'un trafic d'organe s'opérant aux dépens des immigrés clandestins. Le personnage central est originaire d'Afrique du Sud, comme Jerry Masslo dont l'assassinat, la même année, fut à l'origine de douloureuses prises de conscience en Italie. Ces événements tragiques nous sont contemporains. Ils adviennent sans troubler outre mesure notre conscience de (télé) spectateurs et, pour la plupart d'entre nous, la passivité constitue le meilleur refuge. Nos sociétés manqueraient-elles de compassion? Il n'est ici nullement question d'instruire le procès des sociétés occidentales mais seulement de souligner que certains auteurs, qui ont élaboré, à travers leurs écrits, une réflexion nourrie par ces questions, pourraient bien remplir la fonction de ce que nous nommerons des "éveilleurs». Leur texte s'inscrit dans une dimension que l'italien définit comme letteratura civile. 
Publié dans la collection «assolo» des éditions E/O, Ilpadre e lo straniero est un roman difficile à classer. Il rassemble tous les ingrédients nécessaires à l'écriture du roman policier: il y a du mystère, une disparition, un cadavre, des coups de feu, des personnages ambivalents, enfin, des indices apparents sont disséminés mais ils ne conduisent à aucune solution. Car le nouage ne se fait pas et ces ingrédients n'arrivent jamais vraiment à constituer un «impasto». De même qu'il n'y a pas eu véritablement de «nouage», il n'y aura pas de dénouement... Bref, si le lecteur attend qu'on lui raconte une histoire, il verra son attente déçue. Entre "giallo" et «noir», le livre se présente comme un roman policier mais il ne l'est visiblement pas.

C'est, en fait, la rencontre entre deux hommes, qui est au cœur du récit. Tout les oppose - origine, statut social, mode de vie... - mais un même drame les rapproche car tous les deux sont pères d'un enfant handicapé. Le récit s'ouvre dans la cour de l'Institut de pédiatrie, triste microcosme hors du "vrai» monde, où ils conduisent leur fils respectif tous les samedis matins. Pour Diego Marini, simple employé au "Ministero di grazia e giustizia», cette contrainte va devenir peu à peu, un moment privilégié, attendu tout au long de la semaine, et une amitié va naître entre les deux hommes. Avec la disparition improviste du «straniero» (chap. 9), le récit bascule dans le registre du "giallo" (ou du roman noir). Diego Marini se voit sollicité de manière plus ou moins insistante par divers personnages mal identifiés. Policiers? Fonctionnaires des services de sécurité? Espions? On ne le saura pas. Alors que la première partie du récit avait placé la rencontre avec l'autre sous le signe d'une quasi-épiphanie, associée à des expériences et valeurs toujours positives (ouverture, partage, plaisir, solidarité, confiance...), la disparition de l'étranger et le retour en force du "même" (employés et collègues du bureau de Diego, beaux-parents, forces de l'ordre...) voit l'irruption de la violence, de la coercition, de la duplicité, de la trahison...

Ainsi, une fois exclue l'hypothèse selon laquelle De Cataldo aurait voulu nous raconter une histoire policière, nous en étions venue à penser que ce petit roman reposait sur une sorte de jeu d'écriture consistant à inverser le schéma qui associe "même» et amical - d'autant qu' «italiani brava gente» est un axiome toujours en puissance - et «autre» et dangereux. Ce postulat se trouve renforcé par le contexte socio-économique propre à l'Italie, traumatisée par l'afflux des "hordes migrantes" abordant à ses rives depuis les années 90. Ces visions d'exode et de chaos ont été assez relayées par les médias - en particulier entre 1991 et 1993 avec les bateaux d'Albanie pour avoir durablement marqué l'imaginaire social. Dix ans plus tard, le 
traumatisme du onze septembre propage ses ondes de choc jusqu'en Italie. Depuis lors, la figure de l'étranger arabe est associée à celle du terroriste en puissance ${ }^{7}$.

Ce n'est donc pas n'importe quel type d'étranger qui est mis en scène dans le roman de De Cataldo mais un personnage mystérieux provenant d'une partie du monde hautement suspecte. Son origine n'est d'abord pas mentionnée explicitement - même si on devine que Walid vient du Moyen-Orient - enfin, il est fait mention de sa naissance au Liban. On remarque que De Cataldo n'utilise aucun des termes qu'on rencontre couramment dans la presse pour désigner les immigrés arabophones. Faut-il y voir la manifestation d'une décolonisation profonde de son regard $?^{8}$ Un regard qui serait porté sur l'autre dans le respect absolu de son altérité, laquelle n'est pas vue sous le signe de l'infériorité? Diego est fasciné par l'élégance de Walid, son aisance et son savoir-vivre. Bientôt, celui-ci devient son mentor à travers une Rome cosmopolite. La ville s'ouvre à lui, "indigène romain » sur les pas d'un guide allogène, certes, mais plus informé, mieux introduit et mieux "outillé» pour circuler au milieu d'une Babel où lui, Diego, se sentirait étranger. Piazza Vittorio, les bains turcs, la discothèque orientale sont les étapes de ce parcours à la (re)découverte d'une ville qui lui révèle soudain ses doubles fonds, ses arrière-cours, une troisième dimension insoupçonnée.

À cette étape de notre lecture, nous sommes donc tentée de souscrire à l'hypothèse selon laquelle De Cataldo n'entendrait ici nullement nous raconter une histoire mais jouerait de l'inversion des stéréotypes. L'Italien "civile» devient le barbare, face à un étranger "gran signore». Car le degré de savoir-vivre de Walid n'a d'égal, dans le roman, que la vulgarité des collègues de Diego au ministère et la brutalité des policiers. Le texte démontre l'inanité de l'opposition entre "même» et "différent», entre «soi » et "autre» ou indigène et étranger, les deux faisant ici cause commune à travers l'expérience partagée d'une même douleur. Le but pourrait être de créer, dans l'imaginaire du lecteur, un espace de réflexion, au sens optique du terme, une image capturant l'essence de la nature humaine, c'est-à-dire son aptitude à la « condivisione del dolore».

Quando Einstein, alla domanda del passaporto, risponde 'razza umana', non ignora le differenze, le omette in un orizzonte più ampio, che le include e le supera. È questo il paesaggio che si deve aprire: sia a chi fa della differenza una discriminazione, sia a chi, per evitare una discriminazione, nega la differenza?

écrivait Pontiggia en 2000 dans Nati due volte, autre texte mettant en scène un père confronté au handicap de son fils. 
L'amitié qui soude pour un moment les deux destins de Walid et Diego, se manifeste par la solidarité, par une loyauté qui ne passe pas par la parole donnée (genre qui aurait aussi bien pu être de nature mafieuse) mais, audelà de toute expression verbale, par l'approche empathique de la souffrance de l'autre. Cette dimension éthique transparaît dans un momentclé du récit, lorsque Walid enseigne à Diego à souffler sur le visage de son fils autiste, fermé au contact du monde. Ce moment emblématique transporte le récit dans une autre dimension, au seuil du sacré. Mais la magie n'opère pas dans la réalité $\mathrm{du}$ "noir»: malgré le souffle chaud du père, Youssouf, le fils de Walid, meurt et Giacomo, dans les bras de Diego, se détourne, ennuyé. La tragédie de ces deux pères s'accomplit: leur descendance n'est pas viable. On accède alors à une sorte de double fond du récit qui ouvre sur un propos pessimiste sur la transmission. Nous y reviendrons.

Dans ses aspects formels, la narration est construite pratiquement comme un scénario de film ou de téléfilm. Ainsi, la scène d'ouverture du roman - et on cède aisément à la tentation de parler de scène plutôt que de chapitre - plante le décor et les personnages de manière très visuelle:

Diego fumava una sigaretta dopo l'altra in attesa di riprendersi il bambino. Mancavano dieci minuti alla fine della seduta. Tirava un fresco venticello autunnale, e, al centro dello spiazzo sterrato che separava il reparto dei convulsivi da quello dei cerebrolesi, alcuni ragazzi epilettici giocavano a rincorrersi sotto lo sguardo distratto di un'anziana signora intenta a sferruzzare. Due terapiste tentavano di mettere in posizione eretta un bimbetto dalla testa microscopica. Diego aveva già notato quella creaturina disarticolata, che sembrava uno scherzo della natura. Tra sé lo chiamava "il mostrino" 10 .

Le personnage de Walid fait son apparition comme s'il entrait dans le champ de la caméra:

Non vide l'uomo sinché non gli si sedette accanto, dal lato della panchina immerso nell'ombra. Un quarantenne alto e olivastro, profondi occhi neri, un'eleganza che rasentava la ricercatezza ${ }^{11}$.

Au chapitre dix-neuf, la scène du guet-apens nocturne est aussi très visuelle (on la définirait volontiers comme «filmique», voire "théâtrale»): extérieur nuit; des coups de feu claquent, une sorte de chien des Baskerville, "un cane gigantesco, una belva assatanata» (p. 135), se dresse entre Diego et un cadavre sans visage, à demi dévoré...

L'unité de lieu est respectée dans presque tous les chapitres et la plupart sont très courts (quatre ou cinq pages, aux dimensions du feuilleton). Le "décor» est celui de la Rome urbaine et périurbaine. Quelques scènes 
situées à Ostie gardent l'empreinte de souvenirs littéraires placés sous le signe de la mort. Une des scènes les plus réussies, à notre avis, est celle qui manifeste l'absence par la représentation du petit manteau de Youssouf, abandonné sur un banc, dans le jardin désert de la maison d'Ostie (chap. 10).

Rome, chez De Cataldo, est une ville violente mais, dans une époque violente, elle ménage pourtant des «interstices» - ou voies de fuite - inattendus.

Una Roma lussureggiante di crimini e segreti acquattati nell'ombra, piena di interstizi e cul de sac, di luoghi sconosciuti e resi estranei soprattutto dal loro contenuto di umanità: mimica, gergo, gerarchie e relazioni non domestiche, misteri antropologici da decifrare ${ }^{12}$.

comme la décrit Maria Agostinelli, présentant l'ouvrage sur le site internet Railibro. En somme, Rome recèle une plus grande part d'exotisme que le personnage de l'étranger lui-même. Sur les pas de ce dernier, la ville est devenue un espace fantastique offrant à Diego Marini l'opportunité inattendue d'un voyage sur place. Pleine de culs de sac, de doubles fonds, $\mathrm{d}^{\prime}$ "interstizi», la ville lui a permis de "dilatare lo spazio ${ }^{13}$ ", et c'est le détour par cette Rome de l'«autre» - celle des bains turcs, du marché de Piazza Vittorio, de la discothèque orientale - qui a sorti de son enfermement Diego Marini, à l'étroit dans une vie monotone.

En marge de notre lecture, on pourrait poser la question de la reterritorialisation de l'espace urbain dans les grandes villes italiennes et de la façon dont elle est ressentie par les habitants de souche plus ancienne. À quelles dynamiques d'inclusion ou d'exclusion cette reterritorialisation donne-telle lieu? De Cataldo évoque la Piazza Vittorio (chap. 3). Comme tout le quartier de l'Esquilino, qu'un article de L'Espresso en 2000 rebaptisait "Ex-Qui-Lin ${ }^{14}$ » selon la graphie d'un vieil immigré chinois qui, ne parlant pas italien, remplissait dans une phonétique approximative ses mandats postaux, certains quartiers de Rome sont au centre de vives polémiques.

Nous avions souscrit à l'hypothèse d'un jeu d'écriture reposant sur l'inversion des stéréotypes. Nous avons pu évoquer un regard «décolonisé». On demeure, cependant, assez gênée par un exotisme de bazar qui guette derrière la porte... Dans certains tableaux (celui de la danse orientale ou du hammam), on sent l'auteur céder à la fascination des arabesques, certaines scènes évoquant l'orientalisme un peu kitsch du XIX ${ }^{\mathrm{e}}$ siècle. Dans la scène de la visite au hammam (chap. 4), le décor est planté de manière encore une fois très visuelle dès les premières lignes du chapitre: 
Il bagno turco era a qualche metro dal vecchio albergo diurno di via Cavour. Non c'era insegna, ma solo, in cima a tre scalini, un vecchietto con tanto di turbante e un asciugamano bianco sul braccio ${ }^{15}$.

Force est pourtant de remarquer que, dans la Turquie actuelle, le port du turban est à peu près aussi courant que la pratique de la mandoline en Italie. Aussi ce "vecchietto con tanto di turbante» évoque-t-il plus une fantaisie mozartienne qu'un trait de réalisme social ${ }^{16}$. De même, au chapitre six, les paroles que Michel, le gérant Gréco-Arménien de la discothèque, adresse à Diego en guise de bienvenue: "Tu ordina e io eseguo, padrone!» (p. 43) nous apparaissent comme une réplique digne du génie de la lampe d'Aladin. Au rang des "turqueries» ou des "arabesques», on compte encore une scène de danse que Walid nomme "danza del ventre", dénomination triviale qui n'est pas usitée au moyen-orient où on parle de "danse orientale» ou de "danse égyptienne». Cet art complexe aux innombrables figures et variantes régionales est réduit sous la plume de De Cataldo à "una curiosa danza, dalle movenze languide e sensuali ", deux qualificatifs dont le moins qu'on puisse dire est qu'ils ne sont pas vraiment inattendus (p. 38). Ceci étant, c'est néanmoins une véritable fascination qu'exprime l'auteur en consacrant presque tout le chapitre à la danse et à la musique orientales et à la transe étrange qu'elles provoquent.

L'approche de l'altérité conduit Diego à entrer en symbiose avec l'autre mais aussi à évoluer de plain-pied dans un monde qui lui était jusqu'alors fermé. De Cataldo représente ici une expérience interculturelle où se joue ce fameux "métissage culturel», objet de tant de débats en Italie entre 2004 et 2005. Cela ne suffit toutefois pas à faire de De Cataldo un fin connaisseur du moyen-orient. Ainsi, son personnage turc s'exprime en arabe $^{17}$ et Walid, voulant initier son ami italien aux traditions moyenorientales, commande un couscous (p. 38), plat inconnu au Liban mais typique, en revanche, de la cuisine d'Afrique du nord. Le texte n'est donc pas fiable, s'il s'agit d'illustrer de façon documentaire les échanges interculturels entre un italien et un moyen-oriental, mais ce n'est sans doute pas là le but recherché par l'auteur.

Rapporté au contexte socio-politique de l'Italie de 2004, il est probable que le message le plus fort véhiculé par De Cataldo est autre. On rapprochera ici le roman d'un film sorti la même année et traitant d'un sujet proche: Le chiavi di casa, de Gianni Amelio, inspiré du roman de Giuseppe Pontiggia, Nati due volte (2000) ${ }^{18}$. Le réalisateur qui, de film en film, décline les figures d'une même thématique - celle du rapport père- 
fils, des difficultés de la filiation et de la transmission - représente, dans Le chiavi di casa, les retrouvailles entre un père et son fils handicapé qu'il a jusqu'alors tenu à l'écart de sa vie. Dans le même temps, le contexte socioéconomique italien et les médias exaltent la réussite et la performance. La société se met en scène, peuplée de «belloni» riches et puissants. La vie, telle que nous la voyons à travers le filtre de nos écrans, est un reality show. En fait, le spectacle masque une réalité économique moins brillante. Les médias tiennent d'ailleurs deux discours en parallèle qui se contrediraient s'ils entraient en contact. Tandis que les journaux titrent sur la «nouvelle pauvreté» en Italie, qu'on débat du coût de la vie et de l'inflation générée par l'euro, à la télévision défilent $i$ belloni, $i$ ricchi e $i$ vincenti. La mise en scène de soi-même devient une sorte d'impératif susceptible de tenir lieu d'éthique: au fond, tout se résume à far bella figura. Sur fond de néolibéralisme, la compétition gagne tous les domaines: non seulement la vie est un spectacle mais c'est aussi una gara sportiva. L'éthique de l'époque - à certains égards, on ne peut plus étique - commande d'être performant. Dans un contexte de cette nature, poser la question de la place qui revient à ceux qui ne sont ni beaux, ni riches, ni nés pour être des "gagnants" devient quasiment subversif. La question posée ici, à travers les thèmes de la paternité et de la filiation, n'est autre que celle des valeurs à transmettre. Elle n'est pas sans rapport avec celle de la place faite à l'étranger.

Y a-t-il une possibilité véritable de décoloniser assez nos esprits occidentaux pour le recevoir comme "autre" à égalité et non pas comme autre «inférieur» représentant une menace? Il nous semble que c'est cette voie qui s'esquisse dans le roman. Malgré quelques traces d'orientalisme kitsch, ce que nous dit Giancarlo De Cataldo, c'est qu'à travers la «condivisione del dolore», on entre dans une même humanité. Les alter ego «naturels» de Diego, petit fonctionnaire au Ministère de la Justice, n'étant pas ses proches: ni ses collègues et leurs considérations graveleuses après qu'ils l'ont vu en compagnie d'une séduisante amie de Walid; ni l'allié prétendu, Santini, figure ténébreuse et brutale; pas davantage sa propre femme, tenue à l'écart d'une relation d'amitié où elle jouerait le rôle d'une intruse; au bout du compte, c'est bien l'étranger qui s'inscrit naturellement dans l'évidence d'un partage, "[in] quel tempo senza tempo delle loro mute confidenze» (p. 47). Alors s'établit une relation où n'entre aucune forme de prepotenza.

Bien que De Cataldo parle d'optimisme à propos de son roman ${ }^{19}$, nous sommes frappée par la tragédie de ces deux pères "en impasse». Leurs enfants ne sont pas viables et on est au bout d'une chaîne de transmission. Et pourtant, ce sont bien eux: ces pères impuissants et ces fils en état de 
faiblesse, qui sont des garants d'humanité dans un récit traversé de bruit et de fureur. C'est d'ailleurs à son fils, et à personne d'autre, que Diego entend rendre compte de ses actes, après l'échec d'une tentative de sauvetage héroïque ${ }^{20}$.

Le fil logique de la trame pseudo-policière nous échappant, le lecteur finit par se retrouver dans une position qui le rapproche de ces deux enfants autistes, empêchés dans leur décryptage du réel. Le monde extérieur, lieu de l'agitation et source de menaces diffuses, nous devient étranger, comme il l'est pour Youssouf et Giacomino. L'intrigue échappe, l'histoire déborde le cadre du récit: à deux reprises, on croit qu'on va savoir, comme on dit, le «fin mot de l'histoire» mais cette attente est déçue. Une première fois, c'est Santini, "lo sbirro", qui semble près d'expliciter à Diego Marini les tenants et les aboutissants de la disparition de Walid, mais il n'en sera rien. Au dernier chapitre enfin, Walid lui-même, au moment de quitter son ami et de disparaitre dans un autre pays, une autre histoire, offre, par loyauté, de lui révéler son identité véritable et les motifs de ses actes, mais Diego refuse de savoir, lui donnant ainsi la preuve d'une amitié inconditionnelle.

Si abbracciarono commossi. Non riuscivano a trovare le parole adatte. Non ne sprecarono nessuna di inutile o dannosa. Walid era smagrito, insaccato in un cappottino unto, con i cappelli lunghi, ma lo sguardo era limpido, il sorriso mite. Diego salì a bordo del taxi, Walid si mise al volante. Presero la direzione di Firenze. Per un po' si limitarono a fumare una sigaretta dopo l'altra, poi Walid ruppe il silenzio.

"Ti devo delle spiegazioni, amico".

"Non voglio sapere niente".

"Forse è meglio così". (p. 140)

Au terme de la lecture, l'image rémanente d'une génération condamnée à ne jamais se mettre debout, projette une ombre sur le futur qui pourrait découler des choix de l'époque ${ }^{21}$. Dans l'Italie nocturne et violente du roman noir, l'espace est restreint pour le "minorato »: handicapé, immigré, présumé suspect qu'on malmène d'abord (chap. 10: l'enlèvement de Diego par les "sbirri») avant de chercher, éventuellement, à comprendre... Au-delà du roman, l'enfant déficient, l'étranger, l'handicapé mais on pourrait ajouter le quatrième âge et les économiquement faibles détonnent dans le show de l'Italia "razza padrona». De par ses fonctions de juge en Cour d'Assise, De Cataldo ne pouvait manquer d'apercevoir les coulisses du show. Est-ce l'envers du décor qu'il nous donne à voir dans les doubles fonds du récit ou bien un rappel de la substance, sous les paillettes? Il se pourrait, au fond, que l'auteur nous mette ici en garde 
contre la disparition, dans l'Italie contemporaine engagée dans une compétition néolibérale sans états d'âme, d'un sentiment démodé qui est celui de la compassion. On voudrait conclure sur ce point, loin d'être anecdotique, mais, comme nous l'avons dit, le récit est construit de manière très visuelle et l'image de fin appelle un ultime commentaire. Elle n'est pas sans évoquer le final récurrent d'une célèbre bande dessinée française où un "poor lonesome cow-boy», libre de toute attache, s'en va vers le crépuscule. Au dernier chapitre, Walid a réapparu, là où on ne l'attendait pas. Sorte de moderne prince du désert, il voyage léger. Émancipé de sa propre histoire et de son identité (il a de nouveaux papiers), son nom véritable restera inconnu du lecteur comme de Diego. Il n'est pas même aliéné par l'argent puisqu'il renonce à la mystérieuse mallette sauvée par Diego et lui en fait don. Fantasme de liberté, idéal improbable, fata morgana qui apparaît et disparaît, il incarne la figure de l'homme libre qui trace sa route et préserve son intégrité sans accepter de mutilation d'aucune sorte pour rentrer dans la norme, et pour qui «même» et " autre», «normal» et "différent», non seulement n'ont plus de sens, mais sont des catégories inopérantes.

\section{Notes}

1. G. De Cataldo, Romanzo criminale, Torino, Einaudi, Stile Libero, 2002.

2. Voir nos précédents articles: "Voix africaines en Italie», in Exil et migration, Presses universitaires de Caen, Cahiers de la MrSH, octobre 2003, p. 197-212; «Auteurs d'Afrique et lettres italiennes - Quelques réflexions autour de la littérature de la migration », in, «Regards culturels sur les phénomènes migratoires", Babel, $\mathrm{n}^{\circ}$ 11, Université du Sud Toulon-Var, premier semestre 2005, p. 235-254; "La littérature migrante en Italie», Mélanges pour Marie-Hélène Caspar: Littérature italienne contemporaine, Musique, Paris X-Nanterre, CRIX, 2005, p. 591-606.

3. S. Dazieri, La cura del gorilla, Torino, Einaudi, 2001.

4. A. Camilleri, Il ladro di merendine, Palermo, Sellerio, 1996.

5. Voir, dans cet ouvrage, F. Manai, «Extracomunitari protagonisti nella letteratura italiana: il keniota Warùi di Una ignota compagnia di Giulio Angioni».

6. A. Camilleri, Il giro di boa, Palermo, Sellerio, 2003.

7. Dans le cadre d'un cours de licence intitulé «Presse italienne et société» à l'université Paris 3 , nous avons invité nos étudiants à faire une recherche dans quelques quotidiens italiens sur la représentation de l'immigré venu du monde arabe. Il est intéressant de noter que l'appellation change de façon tout à fait tendancieuse au cours des vingt dernières années. Le terme de "Vu' Cumpra'» a d'abord été forgé dans les années quatre-vingts pour désigner les immigrés - souvent Sénégalais ou Marocains - vendeurs ambulants proposant sur les parvis des gares ou sur les plages, lunettes de soleil, boissons gazeuses et autres marchandises. Dans les années 90, l'imminence de l'adhésion à Euroland et les fameux critères de Maastricht conduisent à une sorte de focalisation autour de la question de l'Europe. C'est ainsi que le terme d' «extracomunitari» en vient à désigner tous les migrants provenant d'autres pays que ceux d'Euroland. En même temps, le Maroc étant le pays d'origine du plus grand nombre des immigrés d'Afrique du Nord en Italie, le terme de «marocchino" acquiert alors une acception globalisante pour désigner tout immigré arabophone. Progressivement, le terme de «musulmano» (ou «mussulmano»), tend à s'imposer dans l'usage 
pour désigner ces mêmes migrants arabophones, occultant dans les esprits que beaucoup d'Arabes du Moyen-Orient sont de confession chrétienne. Enfin - et cela est net dans l'après-11-septembre, le terme d' "islamico " prévaut dans les écrits de la presse quotidienne que nous avons analysés.

8. Cette question vient comme en écho, à l'appel qu'Armando Gnisci réitère inlassablement de livre en livre: "Noi europei dobbiamo decolonizzarci da noi stessi» (A. Gnisci, La letteratura italiana della migrazione, Roma, Lilith, 1998, p. 97).

9. G. Pontiggia, Nati due volte, Milano, Mondadori, 2000, p. 42.

10. Il padre e lo straniero, début du premier chap., p. 9.

11. Ibid.

12. M. Agostinelli, «Il padre e lo straniero», $<$ www.railibro.rai.it/recensioni.asp?id=291>

13. Nous nous trouvons ici en résonance avec la réflexion proposée par Matteo Giancotti sur l'œuvre de Tondelli et le sentiment d'enfermement de la jeunesse d'Émilie-Romagne. Voir dans le présent ouvrage M. Giancotti, «Il rombo dell'Emilia paranoica: Altri libertini di Tondelli ».

14. "All'impiegata che controlla un modello di versamento viene la ridarola: "Deve mettere Roma, dopo la via, no Ex Qui Lin. Eppoi che sarebbe 'sto 'qui lin', semmai Esquilino” ", Cristina Mariotti, «L'ondata cinese: Ex Qui Lin», L'Espresso, 24 août 2000.

15. Il padre e lo straniero, p. 23.

16. Nous pensons à la représentation du même lieu qui est montré dans le film de Ferzan Ozpetek, Hammam (1998), où le jeu sur la lumière et la pénombre est empreint d'une grande poésie sans pour autant tomber dans le kitsch.

17. Bien sûr, on pourrait imaginer qu'un turc puisse parler l'arabe mais ce n'est pas le cas général; la langue turque, dont les origines restent assez mystérieuses, n'est pas une langue sémitique et les Turcs ne connaissent généralement de l'arabe que les formules apprises par cœur à l'occasion de la récitation des prières, une connaissance comparable à celle que les catholiques d'avant Vatican II avaient du latin.

18. G. Pontiggia, Nati due volte, Milano, Mondadori, 2000.

19. Dans l'article "Il padre e lo straniero di Giancarlo de Cataldo", consultable sur le site du magazine d'information culturelle en ligne <Pordenonelegge.it>, à la date du 21 février 2005, l'auteur déclarait: «Il racconto ha tratti patetici, aggressivi, disperati, ma si conclude su una nota di speranza. Una speranza che potrà forse essere giudicata impossibile, ma nella quale io continuo, ad onta di tutto, a credere fortemente: la speranza che ciò che la politica, il costume, la cultura e la religione dividono possa essere unito dalla condivisione del dolore, dalla sua serena e laica accettazione».

20. Après avoir échoué dans son entreprise de sauver Walid, Diego téléphone à sa femme en pleine nuit et lui demande de réveiller leur fils: «Attese qualche minuto. Poi sentì il lamento del piccolo disturbato nel suo sonno difficile. "Ho fallito" disse, e riattaccò » (p. 125).

21. L'auteur se refuse pourtant à conclure sur une perspective mortifere: aussi les dernières lignes du récit formulent-elles l'espoir d'un autre enfant que Diego appellerait Youssouf et qui rachèterait «la felicità negata agli altri due e ai loro padri» (p. 143). 